Voix et Images

voixetimages

\title{
Nouvelles voix romanesques
}

\section{Pascal Riendeau}

Volume 38, numéro 3 (114), printemps-été 2013

URI : https://id.erudit.org/iderudit/1018319ar

DOI : https://doi.org/10.7202/1018319ar

Aller au sommaire du numéro

Éditeur(s)

Université du Québec à Montréal

ISSN

0318-9201 (imprimé)

1705-933X (numérique)

Découvrir la revue

Citer ce compte rendu

Riendeau, P. (2013). Compte rendu de [Nouvelles voix romanesques]. Voix et Images, 38(3), 141-146. https://doi.org/10.7202/1018319ar

Ce document est protégé par la loi sur le droit d'auteur. L'utilisation des services d'Érudit (y compris la reproduction) est assujettie à sa politique d'utilisation que vous pouvez consulter en ligne.

https://apropos.erudit.org/fr/usagers/politique-dutilisation/
Cet article est diffusé et préservé par Érudit.

Érudit est un consortium interuniversitaire sans but lucratif composé de l’Université de Montréal, l'Université Laval et l'Université du Québec à Montréal. Il a pour mission la promotion et la valorisation de la recherche. https://www.erudit.org/fr/ 


\title{
R O M A N
}

\author{
Nouvelles voix romanesques \\ $+++$
}

PASCAL RIENDEAU

Université de Toronto

Au cours des dernières années, beaucoup de jeunes auteurs ont publié leur premier ouvrage de fiction dans l'une de ces nombreuses petites maisons d'édition dynamiques fondées depuis le début du Xxle siècle. C'est notamment le cas de quatre des cinq auteurs dont les œuvres sont recensées ici. Toutes mes solitudes! ${ }^{1}$ de Marie-Christine Lemieux-Couture, Charlotte before Christ ${ }^{2} \mathrm{~d}^{\prime}$ Alexandre Soublière, Et au pire, on se mariera ${ }^{3}$ de Sophie Bienvenu, Kuessipan. À toi ${ }^{4}$ de Naomi Fontaine, ainsi qu'Histoires sans Dieu ${ }^{5}$ de Karine Rosso donnent à entendre des voix nouvelles et originales. On peut parier que quelquesuns de ces textes n'auraient pas paru sans une certaine audace de la part des éditeurs.

Premier roman de Marie-Christine Lemieux-Couture, Toutes mes solitudes! raconte un voyage à deux fait "sur le pouce», de Montréal jusqu'en ColombieBritannique. Chri, la narratrice, et son amoureux, Jean, qu'elle surnomme son «Couillon », veulent aller planter des arbres dans l'Ouest canadien. L'idée de départ de ce «roman de la route» ne brille pas par son originalité, mais son intérêt se manifeste ailleurs: d'abord dans l'accumulation d'éléments rocambolesques - ceux qui acceptent de les conduire sont tous étranges ou obsédés - tout au long du périple de plus de quatre mille kilomètres, puis dans les commentaires de Chri portant sur la politique, la langue ou la morale. L'exploration géographique se limite surtout à ce que l'on voit le long de l'autoroute transcanadienne. Chri affirme sa méfiance envers de nombreuses choses, dont les convictions, et elle en profite pour se moquer de Jean, qui a des convictions, mais souhaite que personne ne sache qu'il s'est empiffré chez Mc Do. À ce moment-là, elle précise :

Moi d'abord, j'y crois pas beaucoup, aux convictions en général. Le monde se porterait mieux s'il y en avait moins, de patenteux de convictions. Les -ismes sociaux, les

$$
+++
$$

1 Marie-Christine Lemieux-Couture, Toutes mes solitudes!, Montréal, Les éditions de ta mère, 2012, 303 p.

2 Alexandre Soublière, Charlotte before Christ, Montréal, Boréal, 2012, 217 p. 3 Sophie Bienvenu, Et au pire, on se mariera, Montréal, La Mèche, 2011, 157 p. 4 Naomi Fontaine, Kuessipan. À toi, Montréal, Mémoire d'encrier, 2011, 113 p. 5 Karine Rosso, Histoires sans Dieu, Saint-Sauveur-des-Monts, La Grenouillère, coll. «Migrations», 2011, 117 p. 
religions, le choc des civilisations, la science, Internet, les tsunamis [...]. Or, suffit de les écouter un peu, les gens, pour saisir l'écart entre eux et leurs convictions. [...] Je fais la morale à personne, n'empêche que, la source de l'état névrotique, c'est bien les convictions. (30-31)

À sa question simple, Jean n'a pas obtenu de réponse, c'est-à-dire que le passage cité - même s'il relève plutôt du registre oral - n'est pas donné en discours direct. D'ailleurs, dans ce conflit aussi long que le voyage, la narratrice partage peu ses pensées avec son compagnon, comme s'il n'était pas son égal sur le plan intellectuel. Chri est davantage portée vers un conflit intérieur, une sorte de débat existentiel avec ellemême. Elle dit ne faire la morale à personne en particulier - ce qui n'est pas tout à fait exact -, mais elle développe une série de petites réflexions morales tout au long de son voyage. Justement, dans un épisode où un automobiliste chrétien les menace, elle se sert de la Bible pour le convaincre de ne pas les abandonner au bord de la route.

Le roman contient de nombreux passages dans lesquels la narratrice donne son opinion sur différents sujets. Du début à la fin du récit apparaissent de petits textes en retrait, d'une typographie différente, probablement des extraits du journal intime de Chri, même s'ils ne sont pas datés. En revanche, ils contribuent à la fragmentation d'un récit autrement très linéaire. En voiture, son échange avec le fanatique religieux lui donne l'occasion de réfléchir à la bonté humaine, mais sa méditation improvisée ne la satisfait pas. Elle en ajoute donc une plus abstraite qui n'a aucune incidence directe sur les événements : «Corrections. On ne dit pas : "l'homme est fondamentalement mauvais", ni "l'homme est fondamentalement bon"; ce qu'on peut dire de l'homme, c'est qu'il est pris au dépourvu dans une nuit perpétuelle où il avance nu et aveugle en proie au déchaînement de ses pulsions. Autrement dit, il est à la fois son propre Mal et son propre Bien. ( (191) L'automobiliste suivante, une femme que Chri décrit comme une victime, lui donne également l'occasion de se laisser aller à des digressions morales au milieu de l'épisode qu'elle raconte, puis elle fait une pause afin de mieux comprendre sa réflexion: «Je m'impressionne. Moraliste, c'est un métier? Sûrement que je trouverais ça trop effrayant. Je me rendrais vite compte que les simples dynamiques de couple justifient les grandes guerres et les génocides. » (211)

Par ailleurs, deux Québécois qui traversent le Canada sont nécessairement aux prises avec des interrogations sur la langue française: mauvaise traduction, confusion, incompréhension culturelle, tout y passe. Mais ce qui intéresse surtout Chri, c'est la situation linguistique québécoise :

Faire du sens. On dit ça, parfois. C'est qu'on parle bilingue. Les paranos de la contamination de la langue [...] voudraient nous enfermer dans des condoms académiques... que c'est pernicieux, l'anglais, que ça s'infiltre partout [...]. Notre français est un dialecte sculpté à même l'Amérique. Personne ne pourra nous l'arracher. Et si nous ne parlons pas français, peut-être est-ce parce que nous parlons Québ. (291)

Parler «Québ» ? La proposition peut surprendre, mais ce qui étonne moins, c'est le ton employé par Chri, qui attaque tout de suite ses détracteurs potentiels. Dans l'ensemble, qu'elles soient éthiques ou politiques, les réflexions de la narratrice 
transforment ce récit de voyage aux épisodes assez prévisibles en une expérience qui reprend et développe ce que Thomas Pavel appelle le «long débat axiologique jamais résolu, jamais abandonné ${ }^{6}$ » du roman.

La question de la langue, si elle n'est jamais présentée comme un enjeu ou un sujet de débat dans le premier roman d'Alexandre Soublière, Charlotte before Christ, devient rapidement difficile à éviter. En ce sens, le titre nous indique déjà la place que l'anglais occupera dans ce récit que fait Sacha, un jeune homme au début de son parcours universitaire en biologie, amoureux de Charlotte, qui étudie dans une école de danse. Fils d'une famille aisée, Sacha s'amuse avec ses amis de manière insolite. Le roman s'ouvre d'ailleurs sur une activité à laquelle un petit groupe s'adonne à l'occasion, soit de profiter de l'absence d'un couple ou d'une famille durant une fin de semaine dans le but d'occuper illégalement leur résidence. Toutefois, Sacha insiste sur la prudence: «M'introduire dans la maison d'un inconnu pour quelques jours, boire son alcool, manger sa nourriture, pisser sur les divans, je veux bien, mais je ne tiens pas à avoir un dossier criminel. » (10) Hautain, misogyne, baveux, Sacha est un personnage fortement antipathique. La narration autodiégétique renforce cette impression étant donné qu'elle ne permet pas de créer de distance entre les pensées du narrateur et les actions du personnage. Il en est de même pour la langue qu'il privilégie. Le roman est truffé de mots anglais; il est même parfois écrit dans une sorte de franglais chic. Il ne s'agit pas du bon vieux joual, mais bien d'une façon de s'exprimer incluant des centaines de mots anglais qui vise sans doute à montrer que l'on se trouve au cœur de cette culture jeune et nord-américaine. Cela devient particulièrement évident lorsqu'il est question de musique, de cinéma ou des expériences pornographiques du narrateur. Mais l'anglais est partout: «David et Oli sont trop randoms pour trouver des nouvelles recrues. Les temps sont plus softs qu'avant.» (14) «Elle adore cette brand. Elle a un quelque chose de skate going on. » (68) « Je starte l'épisode à 1 heure p.m. [...] Ce n'est pas un vrai de vrai around the clock.» (153-154) Ces trois exemples - choisis un peu au hasard - montrent que la narration en franglais constitue un élément essentiel de la texture du roman.

Le narrateur quitte parfois ce rôle de jeune insolent lorsqu'il est question de ses problèmes de santé. Souffrant de la maladie de Still, il raconte ses traitements, ainsi que les effets secondaires pénibles et débilitants de cette maladie. Non seulement cette partie témoigne d'une plus grande sensibilité, mais elle montre aussi que Soublière peut avoir un vrai style: «Ma maladie dérive d'un trouble immuno-fou qui décide de fusiller mes articulations saines. Système troublé, confus, dérangé. Il se produit une sorte de sabotage et voilà que l'insurrection interne m'empêche de marcher. » (28) On peut regretter que l'auteur n'ait pas exploité davantage ce filon, d'autant plus que le discours sur la maladie se fait sans complaisance, avec juste assez d'ironie et

$$
++
$$

6 Thomas Pavel, La pensée du roman, Paris, Gallimard, 2003, p. 12. 
d'autocritique. Étonnamment (ou non ?), c'est aussi la partie où le franglais apparaît le moins. Ce court chapitre montre le meilleur de ce jeune auteur qui cherche sans doute à provoquer, mais à qui personne n'a dit que la plus grande subversion ne se trouve peut-être pas là où il pense. On pourrait facilement trouver ce roman insupportable tant le narrateur fait preuve d'immaturité et d'arrogance. De plus, l'histoire d'amour entre deux jeunes dont l'adolescence semble s'étirer et qui se prennent un peu pour "Sid et Nancy» n'a rien de très original. Quant aux puristes, ils ne seront pas les seuls à réagir face à cet envahissement de l'anglais dans le roman. En revanche, ce que Soublière réussit bien, c'est de pousser un peu plus loin la limite de l'acceptable - la conclusion ne rend pas Sacha plus sympathique, au contraire - , du tolérable.

$+$

Et au pire, on se mariera est le premier récit pour adultes de Sophie Bienvenu, elle qui s'était fait connaître avec sa série pour adolescents, ( $k$ ). Encore plus que les deux auteurs précédents, Bienvenu mise sur l'oralité pour forger son style. La narratrice est une adolescente de treize ans, Aïcha Saint-Pierre, qui raconte une histoire qui vient de se produire et dont on apprend les détails au fur et à mesure, mais le récit ne progresse pas toujours de façon linéaire. Reprenant le même procédé que Salinger dans The Catcher in the Rye ${ }^{7}$, roman dans lequel le jeune narrateur, Holden Caulfield, s'adresse à un interlocuteur - un psychologue ou un psychiatre - qui ne répond jamais, Aïcha se livre à une travailleuse sociale ou à une psychologue sans que nous ayons directement accès à la parole de celle-ci. Aïcha a-t-elle été victime des abus sexuels de son beau-père? Baz, le jeune homme qu'elle admire et qu'elle désire, l'a-t-il violée? Qui a commis le meurtre à l'origine de ce drame? Aïcha n'est-elle qu'une pauvre victime ou bien une jeune adolescente mythomane et manipulatrice? Sophie Bienvenu ne donne aucune réponse claire à ces questions, et c'est là une des forces de ce récit, qui aurait pu autrement verser dans la complaisance et le misérabilisme.

La parole d'Aïcha s'impose rapidement. Son expression est directe, colorée, parfois drôle, mais aussi souvent colérique: «Anyway, je suis tout le temps en crisse, y paraît.» (14) L'auteure ne s'est pas contentée de reproduire la parole d'une jeune fille, elle a véritablement inventé un style qui, sans tomber dans l'excès, laisse place à beaucoup d'inventivité : «Ça, j’y crois pas trop, ou alors Dieu, c'est un sale con. Mais bon... Que ce soit le destin, le hasard, Dieu ou un flo extraterrestre plein de cash qui $s^{\prime}$ amuse avec nous comme avec une ferme de fourmis, Baz et moi, on ne s'est pas croisés.» (21) En conflit perpétuel avec sa mère, proche de Mélissa et Johannie, deux prostituées (en réalité, ce sont deux hommes travestis ou des transgenres), cultivant sur le plan sexuel les relations ambiguës avec des hommes plus âgés (un remplace le père, l'autre le grand frère), Aïcha est à la fois une enfant qui veut tout de suite vivre la vie d'une femme et une adolescente sentimentale dont la quête d'identité et une certaine impuissance la poussent à faire des gestes étranges ou discutables, à adopter

$$
++
$$

7 J. D. Salinger, The Catcher in the Rye, New York, Back Bay Books/Little Brown, 2010 (1951), 277 p. 
des comportements risqués. Le pseudo-monologue d'Aïcha est une course, une série d'envolées portées par un souffle, une voix singulière. Ce récit dramatique contient plusieurs passages humoristiques, bouscule quelques idées préconçues et évite presque toujours les discours moralisateurs.

Kuessipan. À toi de Naomi Fontaine explore un territoire que l'on voit trop peu dans la littérature québécoise, celui des villages amérindiens. Louis Hamelin a écrit à propos de Kuessipan qu'il «est aussi différent de la littérature innue qui l'a précédé que Le survenant l'était des romans de la terre québécois ${ }^{8}$ ». Récit aux accents intimistes composé de quelques dizaines de fragments et de petits récits sur la vie des Innus de la Côte-Nord, son fil conducteur tient dans la voix de la narratrice anonyme. Sensible sans être mièvre, Kuessipan contient les observations de la narratrice sur les gens qui l'entourent. Celle qui critique sans juger propose une méditation poétique et existentielle sur la vie des Innus, que ce soit l'homme au tambour, l'arrière-grand-mère morte à cent un ans ou l'amie "plus mince, plus blanche, plus timide» (15) qu'elle. On constate souvent un certain désarroi de la part de la narratrice, notamment à l'égard des jeunes hommes qui ne connaissent pas «ce sentiment masculin de fierté d'être non seulement pourvoyeur, mais aimant envers sa famille» (61), ou encore des filles qui tombent enceintes assez jeunes: «Les filles ne se font pas avorter par ici. Elles endurent, elles survivent. Elles jouissent, quelquefois, des éphémères plaisirs de l'alcool.» (38)

La pensée qui traverse le récit s'éloigne des grands discours et des abstractions, mais elle s'inscrit dans une morale du devoir envers l'autre:

Je n'ai pas le droit.

[...] Pas le droit de laisser tomber ce soir, malgré la fatigue et les pleurs qui me gardent en éveil, en somnolence, en épuisement postnatal.

Je n'ai pas le choix, car je n'ai pas le droit. Les hommes, ils étudient et réfléchissent aux choses surhumaines de l'existence. Ils se cadrent et s'examinent, ils oublient l'essence. [...]

Bien sûr que je n'ai pas le droit d'oublier mon instinct de nomade, sans cesse à la recherche d'un état de grâce. (108)

On voit que la réflexion axiologique est tournée vers la communauté, les ancêtres, la descendance. Le dernier fragment est d'ailleurs entièrement consacré à son fils. À toi : le destinataire explicite du récit, c'est bien le bébé de la narratrice, celui qui a «la peau brune d'un Indien. [...] C'est un vrai Innu, c'est certain.» (110)

$$
++
$$

8 Louis Hamelin, cité sur la quatrième de couverture de Kuessipan. 
D'un registre fort différent, le recueil de nouvelles de Karine Rosso, Histoires sans Dieu, est un projet original qui consiste à reprendre une série d'épisodes de la Bible et à les adapter au monde contemporain. Dans sa très courte postface, Rosso précise qu'elle n'est «ni religieuse ni pratiquante» et qu'elle s'intéresse seulement à «la dimension incroyablement humaine de ces histoires [bibliques] qui se répètent depuis que le monde est monde » (113). Adoptant un style sobre et une langue précise, Karine Rosso s'inspire de la Genèse et de différentes parties du Nouveau Testament afin d'imaginer des histoires où Ėve, la femme de Loth ou Jésus pourraient s'incarner aujourd'hui dans différents lieux des Amériques - du Québec à l'Argentine, en passant par la Californie et le Mexique -, et occasionnellement de l'Europe. Dans ce recueil, on sent que l'auteure a fait preuve de prudence, ne voulant ni ridiculiser les textes sacrés ni encenser leur prétendue vérité, leur sagesse ou leur vertu. Lorsqu'elle glisse du côté de la critique sociale, celle-ci reste peu appuyée. On voit bien çà et là de petites pointes, dont une lancée vers Nicolas Sarkozy - à l'époque où il était ministre de l'Intérieur - , faisant des déclarations incendiaires sur «la racaille» («Exils»), mais cela reste mineur en comparaison de la complexité du récit de Ruth, qui a fui très jeune une Espagne en guerre pour gagner la France. Sans Dieu, les personnages de chacune de ces histoires - qui racontent souvent des conflits terribles où les solutions sont impossibles - n'attendent pas d'intervention miraculeuse. Rien de tout cela n'existe dans un monde où Dieu a disparu, mais où les passions humaines s'expriment encore avec férocité ou grandeur. Un des plus beaux exemples est celui de Sara dans «Piscines», histoire reprise du livre du prophète Isaïe. Mariée au président Abraham Johnson (!), Sara refuse d'accorder à la puissante nation une importance plus grande qu'à ceux qu'elle a enfantés: «Sara était de ces êtres qui se sacrifient au nom de l'amour, mais qui se refusent à sacrifier leur amour au nom de quoi que ce soit, si noble et grand fût-il.» (79)

Dans ces cinq romans et récits fort différents, des problématiques et des thèmes communs reviennent: la langue, l'identité ou la réflexion axiologique, qu'elle soit plus essayistique (Lemieux-Couture) ou intégrée aux personnages et aux types de situations (Rosso). Le travail sur l'oralité est très marqué chez Bienvenu ou LemieuxCouture, et, dans une certaine mesure, chez Soublière. Quant à Rosso et Fontaine, il s'agit plutôt d'intégration d'autres langues, mais dans un souci de précision culturelle pour la première, qui ajoute quelques mots d'espagnol, ou dans un dessein plus anthropologique pour la seconde, qui énumère des mots innus et en explique le sens. Ces nouvelles voix dans le paysage littéraire québécois cherchent à ouvrir d'autres horizons et à explorer les limites de la parole et du romanesque. 